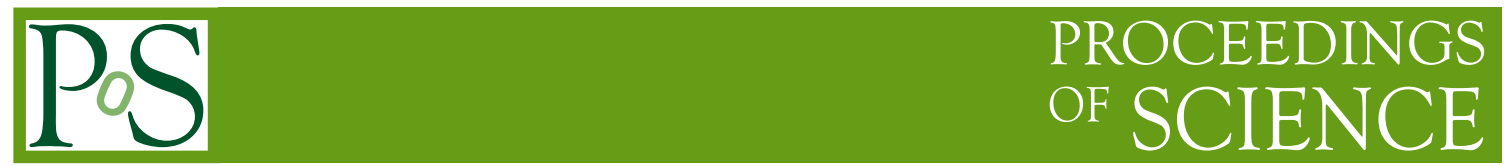

\title{
Light Meson Transition Form Factors on the Lattice
}

\section{Benjamin Owen ${ }^{* a}$, Waseem Kamleh ${ }^{a}$, Derek Leinweber ${ }^{a}$, Ahmed El Bakry ${ }^{a}$ and Peter Moran}

${ }^{a}$ Special Research Centre for the Subatomic Structure of Matter (CSSM),

School of Chemistry and Physics, University of Adelaide, SA 5005, Australia

E-mail: benjamin.owendadelaide.edu.au

Here we present exploratory calculations of the light meson radiative transition moment $\rho \rightarrow$ $\gamma \pi$ from lattice QCD. We observe interesting chiral curvature in the form factor and a definite environment sensitivity in the quark sector contributions. Results are compared with the simple quark model and experiment.

The 30th International Symposium on Lattice Field Theory

June 24 - 29, 2012

Cairns, Australia

${ }^{*}$ Speaker. 


\section{Introduction}

Upon examining the literature for lattice calculations on the radiative decays of the light vector mesons we find some encouraging results with opportunities to make progress. The earliest study by Woloshyn [U] examined these transitions in the context of SU(2) colour and found that his results were comparable to, but systematically smaller than the experimental results. Cristafulli and Lubicz [వ], using SU(3)-colour, were able to extract results that were consistent with experiment, but the large, unphysical pion masses and large $Q^{2}$ values of this study raised questions about their extrapolation to $Q^{2}=0$. Edwards [3] provides a comprehensive calculation of $\rho \rightarrow \pi \gamma$ over a wide range of $Q^{2}$, but for a single, somewhat heavy pion mass. Inspired by the quenched-studies of the

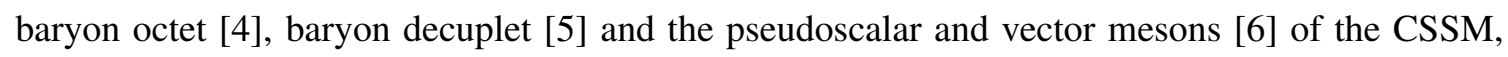
we present a quenched-study of the light-meson transition form factors across a wide range of pion masses to explore the properties of this transition and prepare for a comprehensive calculation in full QCD on large lattices.

\section{Lattice procedure}

For the transition in question, the relevant vertex function [ [ $]$ contains only a single term and takes the form

$$
\left\langle V\left(p^{\prime}\right)\left|J^{\mu}\right| P s(p)\right\rangle=\frac{-i e}{2 m_{V}} F_{V \gamma P s}\left(Q^{2}\right) \varepsilon^{\mu \sigma \alpha \beta} P_{\sigma} q_{\alpha} \varepsilon_{\beta}\left(p^{\prime}, s\right) .
$$

Here $P=p_{1}+p_{2}$ is the total momentum and $q=p_{2}-p_{1}$ is the momentum transfer. We note, as was done in [1], our vertex includes a factor of $m_{V}^{-1}$ to ensure that the form factor $F_{V \gamma P s}\left(Q^{2}\right)$ is dimensionless. We note, this convention was not adopted in [[]].

In our calculation, we use the following ratio of correlation functions to calculate $F_{V \gamma P s}\left(Q^{2}\right)$

$$
R_{v}^{\mu}\left(\vec{p}_{2}, \vec{p}_{1}\right)=\sqrt{\frac{\left(G_{V \rightarrow P s}\right)_{V}^{\mu}\left(\vec{p}_{2}, \vec{p}_{1} ; t_{2}, t_{1}\right)\left(G_{P S \rightarrow V}\right)_{V}^{\mu}\left(\vec{p}_{1}, \vec{p}_{2} ; t_{2}, t_{1}\right)}{\left(G_{V}\right)_{v v}\left(\vec{p}_{2}, t_{2}\right)\left(G_{P S}\right)\left(\vec{p}_{1}, t\right)}} .
$$

Here there is no sum on repeated indices.

Using the vertex defined above, one can show that the ratio is related to $F_{V \gamma P s}\left(Q^{2}\right)$ by

$$
R_{v}^{\mu}\left(\vec{p}_{2}, \vec{p}_{1}\right)=\frac{e}{4 m_{V} \sqrt{E_{V}\left(\vec{p}_{2}\right) E_{P S}\left(\vec{p}_{1}\right)\left(\frac{\left(p_{2 v}\right)^{2}}{m_{V}^{2}}-g_{v v}\right)}}\left|\Upsilon_{v}^{\mu}\left(p_{2}, p_{1}\right)\right| F_{V \gamma P s}\left(Q^{2}\right),
$$

where

$$
\Upsilon_{v}^{\mu}\left(p_{2}, p_{1}\right) \equiv \varepsilon^{\mu \sigma \alpha \beta}\left(g_{v \beta}-\frac{1}{m_{V}^{2}} p_{2 v} p_{2 \beta}\right)\left(p_{2 \sigma} p_{1 \alpha}-p_{2 \alpha} p_{1 \sigma}\right) .
$$

Given a choice of momentum for the final and initial states, we are able to calculate the correlation functions and so extract a value for $F_{V \gamma P S}\left(Q^{2}\right)$. A convenient choice, which we utilise here, is to have one of the particles stationary. Importantly, we still have the freedom to choose which of the particles is stationary. This choice in kinematics has the nice feature that it provides us with two distinct values of $Q^{2}$ for a given three-momentum insertion. 


\section{Calculation Details}

The exploratory calculation was carried out on a small ensemble of 75 quenched gauge field configurations, calculated using an $\mathscr{O}\left(a^{2}\right)$ mean-field improved Luscher-Weisz plaquette + rectangle gauge action and fat-link irrelevant clover (FLIC) Dirac operator for the fermions. These lattices are $20^{3} \times 40$ with lattice spacing $a=0.128 \mathrm{fm}$.

We use eight $\kappa$-values giving values for $m_{\pi}$ ranging from $830-300 \mathrm{MeV}$ and hold the strange quark mass fixed with $m_{s \bar{s}}=697 \mathrm{MeV}$. Quark propagators are calculated using smeared sources at $\mathrm{t}=8$. Three-point propagators are calculated using the sequential-source technique where we hold the momentum of the current fixed. For the vector current we use an $\mathscr{O}(a)$-improved FLIC conserved vector current with current insertion at $t=14$. Further details for these ensembles can be found in [四, 回]

\section{Accessing $F_{V \gamma P S}$ at $Q^{2}=0$}

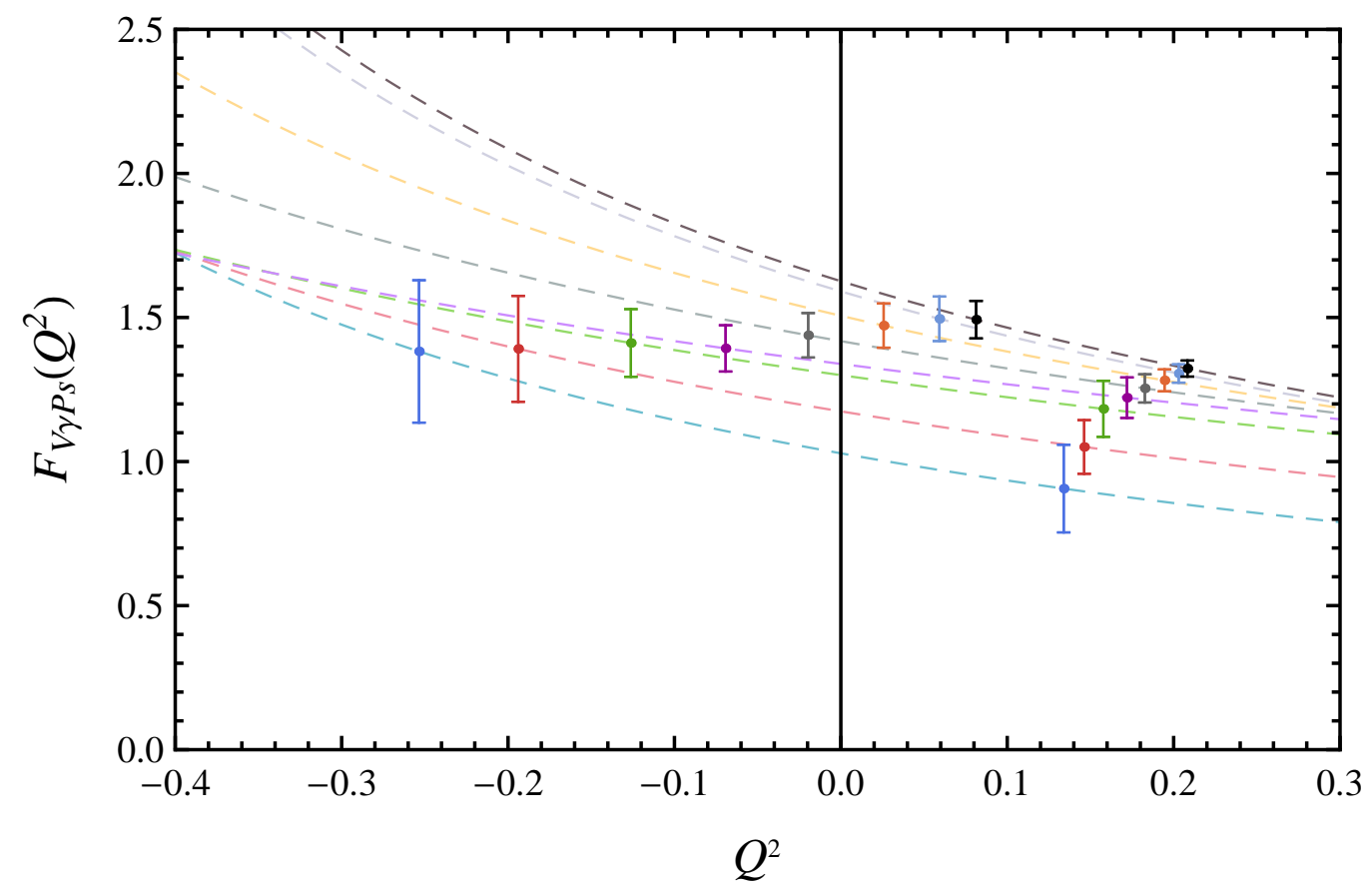

Figure 1: Comparison of monopole fits to each pair of $F_{V \gamma P s}\left(Q^{2}\right)$ for the $\rho \rightarrow \gamma \pi$ transition. Each curve corresponds to a different value of $m_{\pi}^{2}$ with values decreasing from top to bottom.

The vector dominance model suggests that the form factor should exhibit a monopole behaviour at low $Q^{2}$. In [0] vector dominance was found to agree poorly with the data, but we note that this may be due to the large values of $Q^{2}$ investigated resulting from their small lattice volume. In contrast to this, the results of Edwards in [3] , which examined the rho transition over a large range of $Q^{2}$, certainly support the vector dominance hypothesis. As a result, we choose to fit the transition data to

$$
F_{V \gamma P s}\left(Q^{2}\right)=\left(\frac{\Lambda^{2}}{\Lambda^{2}+Q^{2}}\right) F_{V \gamma P s}(0)
$$


in the process of determining $F_{V \gamma P S}(0)$. Figure 1 highlights the $Q^{2}$ dependence for $\rho \rightarrow \pi \gamma$ where the dashed lines are the monopole fits for each value of $m_{\pi}^{2}$ considered.

\section{Quark sector Results}
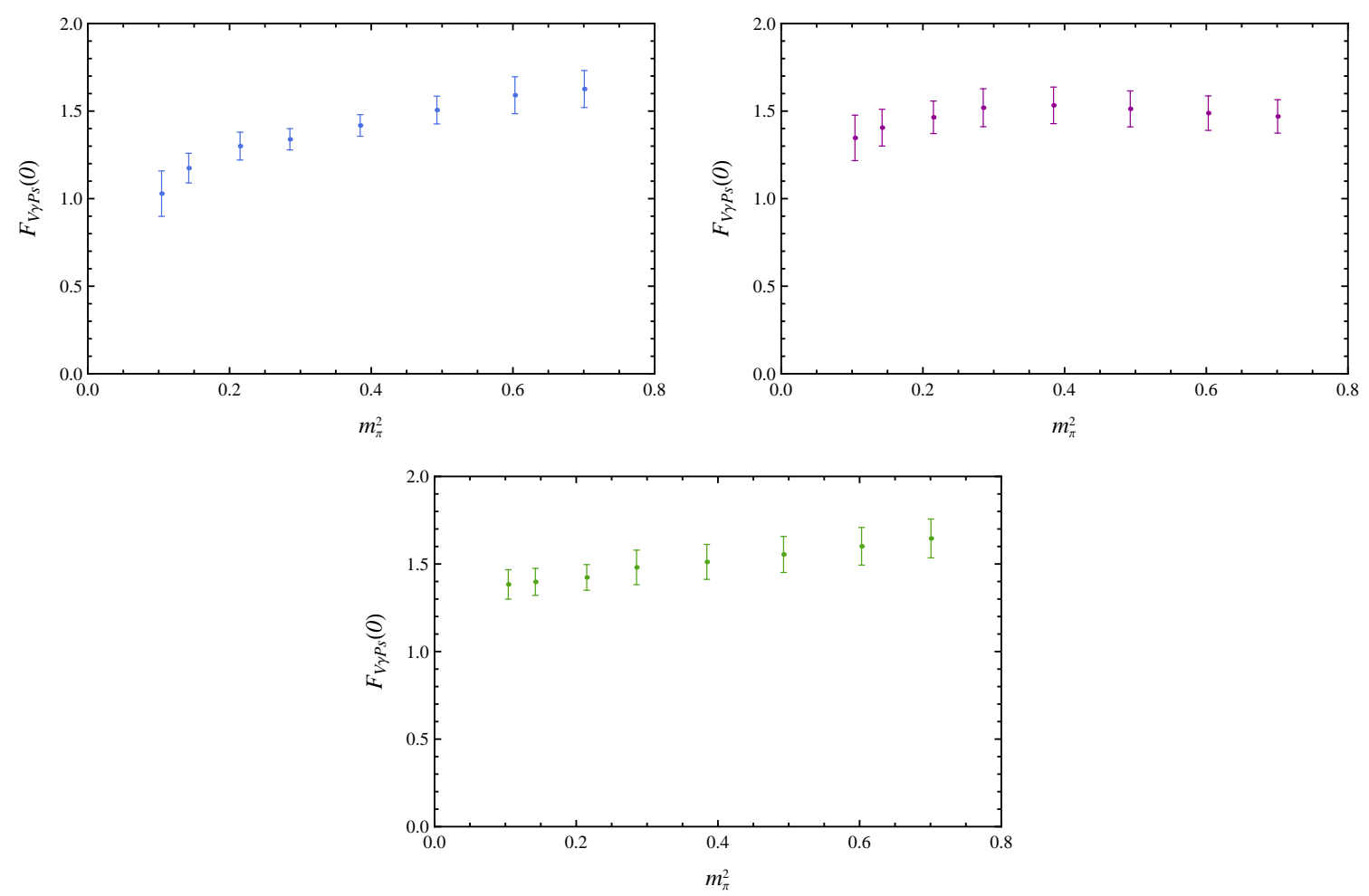

Figure 2: Results for $F_{V \gamma P S}(0)$ for each quark sector with unit charge quarks. The upper-left plot is for $u$ or $d$ quarks in $\rho \rightarrow \pi \gamma$, the upper-right plot is for $u$ or $d$ quark in $K^{*} \rightarrow K \gamma$ and the lower plot is for the $s$ quark in $K^{*} \rightarrow K \gamma$. Note how the light $u$ and $d$ sectors differ between the $\rho$ and $K^{*}$ transitions indicating that the light quark is sensitive to the presence of the strange quark.

By setting the electric charge of one quark sector to unity and the others to zero, one can resolve the contribution of each quark flavour to the form factor. Fig. 2 details the quark sector results for $F_{V \gamma P s}(0)$ as a function of quark mass. Comparing the light $u$ or $d$ quark sector results between the $\rho$ and $K^{*}$, we can see that there is a definite sensitivity to the presence of the strange quark. The curvature exhibited by the light sector in the $\rho$ is significantly reduced in the $K^{*}$.

\section{Meson Form Factor Results at $Q^{2}=0$}

To calculate $F_{V \gamma P s}(0)$ for the hadron in question, we sum the relevant quark sector contributions weighted by the charge of each quark. Having combined the relevant quark sector results, we now examine the transition form factors of the hadrons in Fig. 3. As a comparison, we include simple quark model predictions which are summarised in the review by O'Donnell [8]. We also include experimental results for the transition form factors which we calculate from the decay widths using 

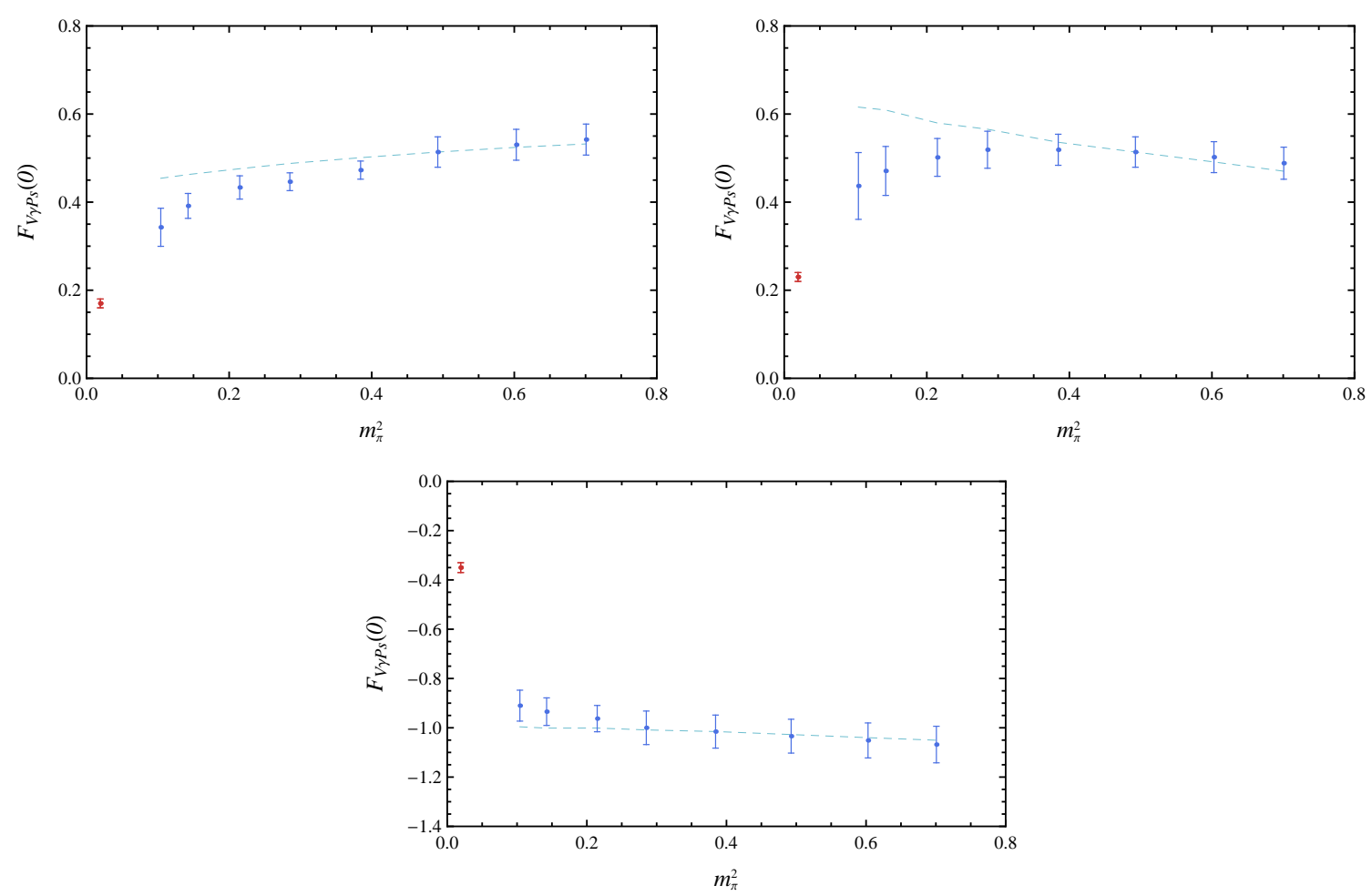

Figure 3: Results for the hadron transition moment $F_{V \gamma P s}(0)$. The upper-left plot is the transition $\rho \rightarrow \pi \gamma$, the upper-right is $K^{* \pm} \rightarrow K^{ \pm} \gamma$ while the bottom plot is in $K^{* 0} \rightarrow K^{0} \gamma$. The data points for $m_{\pi}^{2}>0.1 \mathrm{GeV}^{2}$ are our lattice results, the dashed curve is the quark model predictions while the left-most data points are the experimental results evaluated from the corresponding decay widths and branching fractions.

the expression

$$
\Gamma_{V \rightarrow P s \gamma}=\frac{1}{3} \alpha \frac{|\vec{q}|^{3}}{m_{V}^{2}} F_{V \gamma P s}(0)^{2}
$$

In all three cases, our results display a quark mass trend towards the experimental measurements. It is interesting that for each hadron, all have larger magnitudes at heavy masses and exhibit slight curvature as we approach the physical limit. Of course the curvature displayed at the lightest masses will reflect the associated quenched chiral effective field theory. We intend to follow up this calculation with a full QCD simulation and employ our correlation matrix techniques [Q]. A comparison between our results and those of the quark model show good agreement in the heavy quark regime. As we move towards the physical point, we see that the data deviates from the quark model result due to the onset of the chiral curvature. In particular, for the case of the $K^{* \pm} \rightarrow K^{ \pm}$ transition, we find that the curvature in the data changes direction to that suggested by the quark model indicating that there may be significant meson cloud effects present that are unaccounted for in the quark model. It will be interesting to explore these effects with the inclusion of dynamical fermions. 


\section{Conclusion}

In this work we have calculated the transition moment for the radiative decays of vector mesons. Interestingly we see chiral curvature and a definite environment sensitivity of the quark sector contributions. Having established the framework it now stands to explore this physics in full QCD. It will also be interesting to implement this analysis on the radiative transitions of nucleon resonances [ㅁ], ㅁ], ㅁ]].

\section{References}

[1] R. M. Woloshyn, Vector Meson Radiative Decay In Lattice QCD, Z. Phys. C 33, 121 (1986).

[2] M. Crisafulli and V. Lubicz, A Lattice study of electromagnetic decays of vector mesons, Phys. Lett. B 278, 323 (1992).

[3] R. G. Edwards [Lattice Hadron Physics Collaboration], Electromagnetic hadronic form-factors, Nucl. Phys. Proc. Suppl. 140, 290 (2005) [hep-lat/ 0409119 ].

[4] S. Boinepalli, D. B. Leinweber, A. G. Williams, J. M. Zanotti and J. B. Zhang, Precision electromagnetic structure of octet baryons in the chiral regime, Phys. Rev. D 74, 093005 (2006) [hep-lat/0604022].

[5] S. Boinepalli, D. B. Leinweber, P. J. Moran, A. G. Williams, J. M. Zanotti and J. B. Zhang, Precision electromagnetic structure of decuplet baryons in the chiral regime, Phys. Rev. D 80, 054505 (2009) [hep-lat/0902.4046].

[6] J. N. Hedditch, W. Kamleh, B. G. Lasscock, D. B. Leinweber, A. G. Williams and J. M. Zanotti, Pseudoscalar and vector meson form-factors from lattice QCD, Phys. Rev. D 75, 094504 (2007) [hep-lat/0703014].

[7] M. Chemtob, E. J. Moniz and M. Rho, Deuteron Electromagnetic Structure at Large Momentum Transfer, Phys. Rev. C 10, 344 (1974).

[8] P. J. O’Donnell, Radiative decays of mesons, Rev. Mod. Phys. 53, 673 (1981).

[9] B. Owen, W. Kamleh, D. Leinweber, M. S. Mahbub, B. Menadue, Correlation matrix methods for excited meson form factors in Full QCD, PoS LATTICE 2012 (2012) 173.

[10] S. Nozawa and D. B. Leinweber, Electromagnetic form-factors of spin 3/2 baryons, Phys. Rev. D 42, 3567 (1990).

[11] D. B. Leinweber, T. Draper and R. M. Woloshyn, Decuplet baryon structure from lattice QCD, Phys. Rev. D 46, 3067 (1992) [hep-lat/9208025].

[12] D. B. Leinweber, T. Draper and R. M. Woloshyn, Baryon octet to decuplet electromagnetic transitions, Phys. Rev. D 48, 2230 (1993) [hep-lat/9212016] . 